\title{
Study of the MTF of a MWIR T2SL focal plane array in IDDCA configuration
}

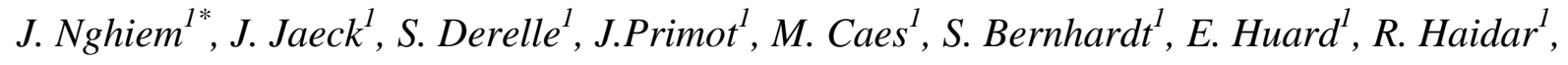 \\ L. Höglund ${ }^{2}$,E. Costard ${ }^{2}$, J.B. Rodriguez ${ }^{3}$, P. Christol ${ }^{3}$, I. Ribet-Mohamed ${ }^{1}$ \\ ${ }^{1}$ ONERA, Chemin de la Hunière, F- 91761 Palaiseau, France. \\ ${ }^{2}$ IRnova AB, Electrum 236, SE-164 40 KISTA, Sweden \\ ${ }^{3}$ IES, Univ. Montpellier, CNRS, F-34000 Montpellier, France
}

Keywords: MTF, T2SL, FPA, MWIR, IDDCA, CSIG.

*Corresponding author: $\quad$ Tel.: $(+33) 180386360$;

E-mail address: jean.nghiem@onera.fr

Highlights:

- $\quad$ MTF of a MWIR T2SL FPA has been measured in a Integrated Detector Dewar Cooler Assembly (IDDCA)

- Non-intrusive access to the pixel size deduced from the MTF measurement.

- Influence of vibrations has been investigated on and the upper boundary of their impact has been studied.

Abstract: Type-II InAs/GaSb superlattice (T2SL) has recently matured into a commercially available technology addressing both MWIR and LWIR spectral domains. As the prerequisites such as Quantum Efficiency (QE) and dark current were met, more advanced figures of merits related to the ElectroOptic (EO) system as a whole can now be studied to position this technology. In this paper, we focus on modulation transfer function (MTF) measurements. Knowing the MTF of a detector is indeed of primary importance for the EO system designers, since spatial filtering affects the system range. We realized MTF measurements on a 320x256 MWIR T2SL FPA provided by IRnova, using a Continuously Self Imaging Grating (CSIG). The advantage of this experimental configuration is that no high performance projection optics is required thanks to the self-imaging property (known as Talbot effect) to project a pattern with known spatial frequencies on the photodetector. Besides, the pattern being propagation invariant, alignment is easier and the bench does not require exact knowledge of the focal plane distance to operate, opening measurement in Integrated Detector Dewar Cooler Assembly (IDDCA) configuration. Extracted from measurements, the pixel size is $26 \mu \mathrm{m}$ for a pitch of $30 \mu \mathrm{m}$. 


\section{I) Introduction}

The modulation transfer function (MTF) of a detector is an advanced figure of merit (FoM). Known for its versatility, it can be exploited in many different ways. On one hand, system designers will want it to anticipate pixel filtering and then optimize their system global performance accordingly. On the other hand, technologists can make use of the MTF as a means to probe the quality of the technological process and intrinsic properties of the material such as electronic diffusion length. There are quite many challenges when attempting to measure an operational detector MTF in the infrared domain (IR). First, the trend of smaller pixels means the diffraction limit and deconvolution become major concerns. As a result, constraints are harsher on any projection optics used in measurement benches. They have to reconcile low chromatism and high aperture while operating within a relatively large spectral band. Such attributes are difficult to achieve at the same time or at a prohibitive cost. Second, IR FPA are likely to be cooled. It also means that access to the FPA is limited. Last, operational IR focal plane arrays are often packaged as Integrated Detector Dewar Cooler Assembly (IDDCA), so the f-number is set by the designer. Our solution to address the three aforementioned issues is a MTF measurement bench exploiting the self-imaging panchromatic property of gratings to project a high resolution periodic pattern on the FPA. It requires neither high quality projection optics nor demanding alignments for the detector MTF to be measured. The type-Il superlattice (T2SL) FPA has been chosen to illustrate our MTF measurement technique since no such characterization has been made so far on theT2SL technology integrated in a cryocooler, that is to say in an operational packaging. Indeed, T2SL have shown promising prospects thus far [1-8]. Thus, it is a suitable candidate for high performance infrared imaging applications in which the MTF measurement is demanded.

In this paper we present the MTF measurements of a $30 \mu \mathrm{m}$ pitch $320 \times 256$ MWIR type-II superlattice infrared Focal Plane Array sealed in a cryocooler. We first present the bench principle and how it is designed. The second part of this paper is dedicated to the vibration study, as they can strongly affect the MTF. The use of accelerometers combined with low integration time measurements allows distinguishing the vibration effect from other contributions on the measured MTF.

\section{II) The measurement bench}

\section{a) Bench description}

The bench aims to measure the MTF ${ }_{\text {detector }}$ of the IDDCA, that is to say the filtering effect due to the pixel. The principle of the bench is to project a pattern with known specific spatial frequencies on the FPA. The measured image is then compared to the theoretical projected pattern in order to extract the MTF. We assume that the pixels of the FPA are uniform so our measurement leads to measuring the MTF of the average pixel. This hypothesis is perfectly 
acceptable because the T2SL has shown thus far promising uniformity [9-10]. The bench is described in Figure 1Erreur! Source du renvoi introuvable. A $1200 \mathrm{~K}$ blackbody is used as the IR source. The size of the projected image of the blackbody having a filtering effect, a $500 \mu \mathrm{m}$ pinhole has to be added to avoid that spatial frequencies are filtered out before reaching the Continously Self Imaging Grating (CSIG), the core of the bench. The CSIG has been developed at ONERA for 20 years [11-14]. It diffracts an engineered specific number $\mathrm{N}$ of orders, generating a pattern that is propagation invariant and achromatic. The frequencies contained in the projected pattern are known by design. Therefore, the MTF is determined by retrieving the amplitudes in the image corresponding to these spatial frequencies. Consequently, the MTF data will be measured on a set number of spatial frequencies, as many as the CSIG excited. The advantages of this CSIG based MTF measurement bench are twofold. First of all, alignment is not critical anymore thanks to the high angular tolerance of the CSIG [15-16]. Besides, the propagation invariant property allows to properly measure the MTF without knowing the exact position of the FPA is a boon when measuring in IDDCA configuration, as defocus is a known issue that can severely deteriorate the measured MTF [17]. It also means that high quality optics are no longer needed as well, releasing constraints tremendously.



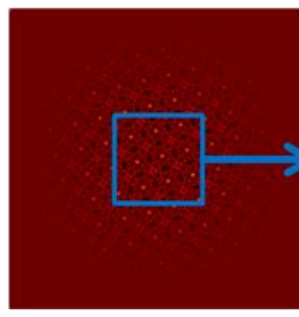



Projected pattern

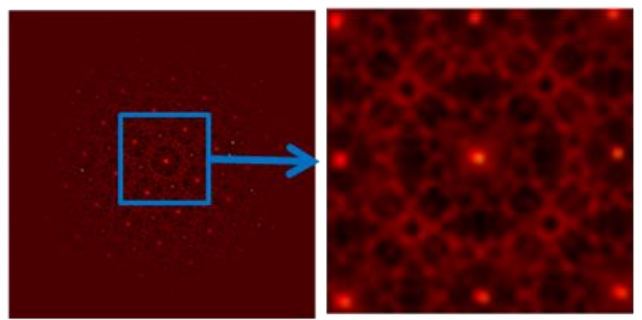

Measured image

Figure 1 - MTF measurement description. On top left, the original setup. On the top right, the principle of the bench. The idea is to compare the theoretical projected pattern with the image measured. 3 distinctive contributors can be distinguished in the figure : the bench, the CSIG and the IDDCA. The offset has been substracted (with the shutter closed) but no non-uniformity correction has been applied. Projected (theorical) and measured images are illustrated as well. 
The $\mathrm{MTF}_{\text {total }}$ measured is not the $\mathrm{MTF}_{\text {detector }}$ alone. It comprises the packaging of the FPA, but also any contributor between the blackbody and the FPA. As a consequence, the model has to take into account the surrounding of the detector.

\section{b) The bench design}

The total measured Transfer Function (TF) can be written as the product of the TFs assigned for each contributor as illustrated on Fig.1. We can first split the global TF into 3 main contributors : the TF of the CSIG, the TF bound to the bench itself, the TF due to the full IDDCA, with $v_{x}, v_{y}$ being the spatial frequencies along $x$ and $y$ axes of the detector:

$$
T F_{\text {total }}\left(v_{x}, v_{y}\right)=T F_{C S I G}\left(v_{x}, v_{y}\right) \times T F_{I D D C A}\left(v_{x}, v_{y}\right) \times T F_{\text {bench }}\left(v_{x}, v_{y}\right)
$$

The CSIG selects spatial frequencies (288 in our case, with the maximum frequency being $\left.51 \mathrm{~mm}^{-1}\right)$. As such, it is regarded as an TF made of a sum of Diracs functions $\delta\left(v_{x}, v_{y}\right)$. These Diracs are weighed because the real object slightly strays from the perfect mathematical object (binarisation effects).

$$
T F_{C S I G}\left(v_{x}, v_{y}\right)=\sum_{i=1}^{288} c_{i} \delta\left(v_{x_{i}}, v_{y_{i}}\right)
$$

With $\mathrm{c}_{\mathrm{i}}$ being weight assigned to a given Dirac function.

The pinhole can be considered as a low filter pass filter defined by its diameter $\varphi_{\text {pinhole. }}$ It corresponds to an Airy disk function (named Airy $\left(v_{x}, v_{y}\right)$ here) and a TF collimator $_{\text {as well (as its }}$ focal length will define the size of the image of the pinhole, which will define in return the cutoff frequency of the pinhole).

$$
T F_{\text {bench }}\left(v_{x}, v_{y}\right)=\operatorname{Airy}\left(v_{x}, v_{y}\right) \times T F_{\text {collimator }}\left(v_{x}, v_{y}\right)
$$

The $\mathrm{TF}_{\text {IDDCA }}$ can be split into a $\mathrm{TF}_{\text {idealpixel, }}$ that describes the perfect behavior of a pixel. and a $T_{F}$ tech. The perfect pixel can be modelized by a $2 D$ sinc with characteristic lengthes $a_{x}$ and $a_{y}$. $\mathrm{TF}_{\text {tech }}$ assumes that every effect altering the $\mathrm{TF}_{\text {idealpixel }}$ can be regrouped into a single $2 \mathrm{D}$ Gaussian function with independent parameters $d_{x}$ and $d_{y}$ :

$$
T F_{I D D C A}\left(v_{x}, v_{y}\right)=T F_{\text {idealpixel }}\left(v_{x}, v_{y}\right) \times T F_{\text {tech }}\left(v_{x}, v_{y}\right)
$$

With :

$$
T F_{\text {idealpixel }}\left(v_{x}, v_{y}\right)=\operatorname{sinc}\left(v_{x}, a_{x}\right) \times \operatorname{sinc}\left(v_{y}, a_{y}\right)
$$

And : 


$$
T F_{t e c h}\left(v_{x}, v_{y}\right)=e^{-\frac{\left(\pi v_{x} \delta_{x}\right)^{2}}{2}} \times e^{-\frac{\left(\pi v_{y} \delta_{y}\right)^{2}}{2}}
$$

The purpose of the dimensioning phase detailed in [15] is to ensure that $T F_{\text {bench }}\left(v_{x}, v_{y}\right)$ becomes negligible to the rest of the model (ie being close to 1). We can consider that the full TF can be written as :

$T F_{\text {total }}\left(v_{x}, v_{y}\right)=\sum_{i=1}^{288} c_{i} \delta\left(v_{x_{i}}, v_{y_{i}}\right) \times \operatorname{sinc}\left(v_{x}, a_{x}\right) \times \operatorname{sinc}\left(v_{y}, a_{y}\right) \times e^{-\frac{\left(\pi v_{x} \delta_{x}\right)^{2}}{2}} \times e^{-\frac{\left(\pi v_{y} \delta_{y}\right)^{2}}{2}}$

The Modulation Transfer Function (MTF) corresponds then to the modulus of the Transfer Function :

$$
\operatorname{MT} F_{\text {total }}\left(v_{x}, v_{y}\right)=\left|T F_{\text {total }}\left(v_{x}, v_{y}\right)\right|
$$

Our measurement is in $2 \mathrm{D}$ as shown in Figure 2. We chose to represent it as a 1D measurement using the variable $v=\sqrt{v_{x}^{2}+v_{y}^{2}}$. Since the diagonal of the pixel also has a filtering effect, there is expected dispersion on the displayed results.
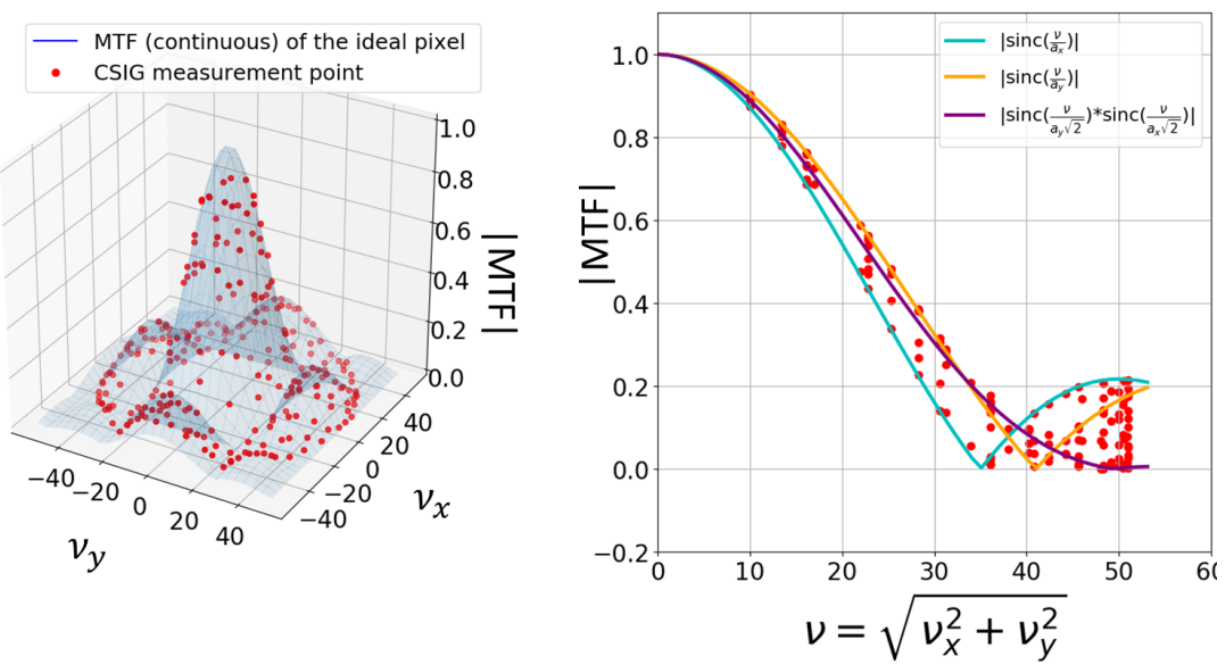

Figure 2 - Data representation of the MTF as a function of spatial frequencies along $x$ and $y$ axes on the left (2D measurement) on an ideal 2D sinc (simulated) with the spatial frequencies sampled by the CSIG (simulated as well). On the right, the MTF representation we adopt using $v=\sqrt{v_{x}^{2}+v_{y}^{2}}$. There is an expected dispersion because of the way we represent the data.

\section{c) Results}

The MTF has been successfully measured as shown in Figure 3 on a T2SL MWIR manufactured by IRnova with an integration time of $6 \mathrm{~ms}$ and a framerate of $60 \mathrm{~Hz}(300$ images averaged with an oversampling factor of 5 in $x$ and $y$ axes). It is possible to extract 
the pixel size without performing fits. Indeed, $\operatorname{MTF}_{\text {total }}\left(v_{x}, v_{y}\right)$ only cancelled by the $2 \mathrm{D}$ sinc at the cutoff frequencies $v_{x}, v_{y}$ due to the pixel filtering (in $x$ and $y$ axis respectively) with:

$$
v_{c x}=\frac{1}{a_{x}} \text { and } v_{c y}=\frac{1}{a_{y}}
$$

$v$ being the cutoff frequency, $a_{x}, a_{y}$ the pixel size in $x$ and $y$ direction.

Since there is a change of sign of $\mathrm{TF}_{\text {total }}$ around the cutoff, it is more reliable to look for a phase shift in the TF phase (that contain the sign information) rather than searching for the zero of the function in the MTF. Figure 3 shows that the phase allows to estimate more reliably the cutoff, thus the pixel size. A $39 \mathrm{~mm}^{-1}$ cutoff has been determined, corresponding to a $26 \mu \mathrm{m}$ pixel size, in agreement with the fill factor designed by IRnova.. By fitting the data with the model with $a_{x}, a_{y}, \delta_{x}, \delta_{y}$ as fit parameters, best agreement is obtained for $a_{x}=a_{y}=$ $26 \mu \mathrm{m}$ and $\delta_{x}=\delta_{y}=10 \mu \mathrm{m}$.


Figure 3 - MTF measurement and fit with a model. On the left, the MTF as a function of the spatial frequency, on the right, the phase of the TF as a function of the spatial frequency.

\section{d) Summary}

We successfully performed the MTF measurement in an IDDCA configuration. The advantage of our measurement bench is that it can be adapted in order to measure other infrared technologies. As a bonus, the measurement bench gives access to the pixel size, using the phase of the MTF as a guideline. However, to successfully fit our measurements by a model, we had to add a 2D Gaussian model, with $\delta_{x}$ and $\delta_{y}$ parameters of $10 \mu \mathrm{m}$ to the ideal 2D sinc. This needs to be investigated as the T2SL studied has been confirmed by IRnova as deep etched. As a result, these parameters are unlikely to be diffusion lengthes. We suspected this filtering effect to be due to the vibrations of the cryocooler. This led us to study the effects of the vibrations on our measurement. 


\section{III)Vibration study}

\section{a) Vibration restitution}

As the compressor of the cryocooler functions, it generates vibrations that are likely to be transmitted to the FPA. As the blur due to vibrations may deteriorate the MTF, vibrations on the measurement bench have been estimated by using the data used for the MTF measurement. Another MTF measurement has been made, this time with $3 \mathrm{~kg}$ weight added. The pattern of the vibrations on both measurements is reported in Figure 4. With $3 \mathrm{~kg}$ weight, since the $Y$ axis is constrained, the energy due to vibrations has to be dissipated mainly on $X$ axis, thus the increase on $X$ axis. As we used 300 for each position of the CSIG, it is possible to estimate shifts between each image. We used the first image as the reference. We rely on the fact that the spatial frequencies excited by the CSIG are sparse as seen in Fig.2. It allows determining the shift between a given image and the reference by estimating the phase shift between the Fourier transform of those images.
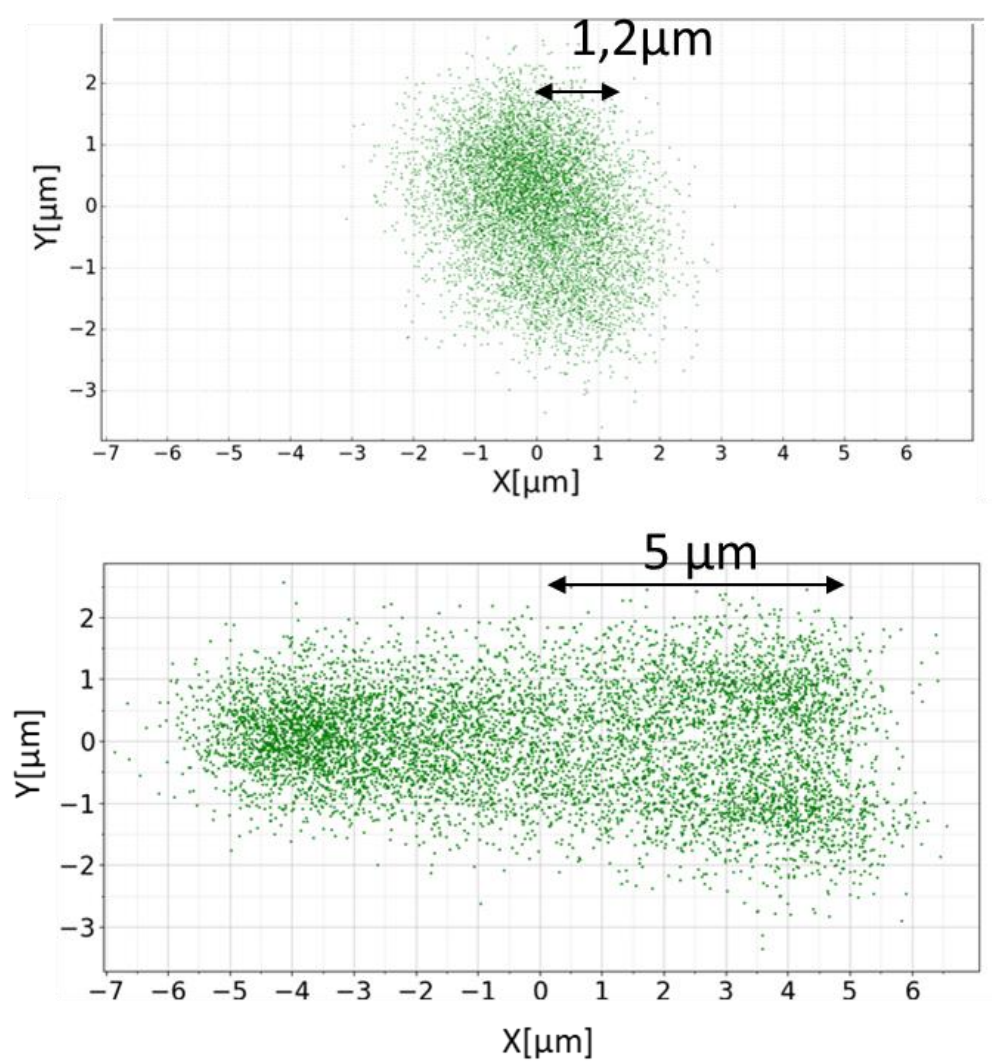

Figure 4 - Vibration pattern restitution. Top figure corresponds to the vibration estimated from the MTF measurement without the $3 \mathrm{~kg}$ weight on the cryocooler, bottom is with the weight. By adding $3 \mathrm{~kg}$ on the cryocooler, the pattern becomes different. One can notice that the $\mathrm{Y}$ axis (constrained because of the mass on the cryocooler) did not drastically change while the $X$ axis amplitude of the pattern has. 
The shape of the pattern has changed from the two setups. As a consequence, the MTFs are shown accordingly in Figure 5. The MTF measurements are different and the fit model shows that the $x$ axis has been deteriorated, which is coherent with the fact that the width of the pattern also increased on the $x$ axis. This means that vibrations can affect the MTF measurement and have to be studied in order to have their impact on the MTF measurement evaluated. This is why we chose to add accelerometers on the bench for the next measurements.
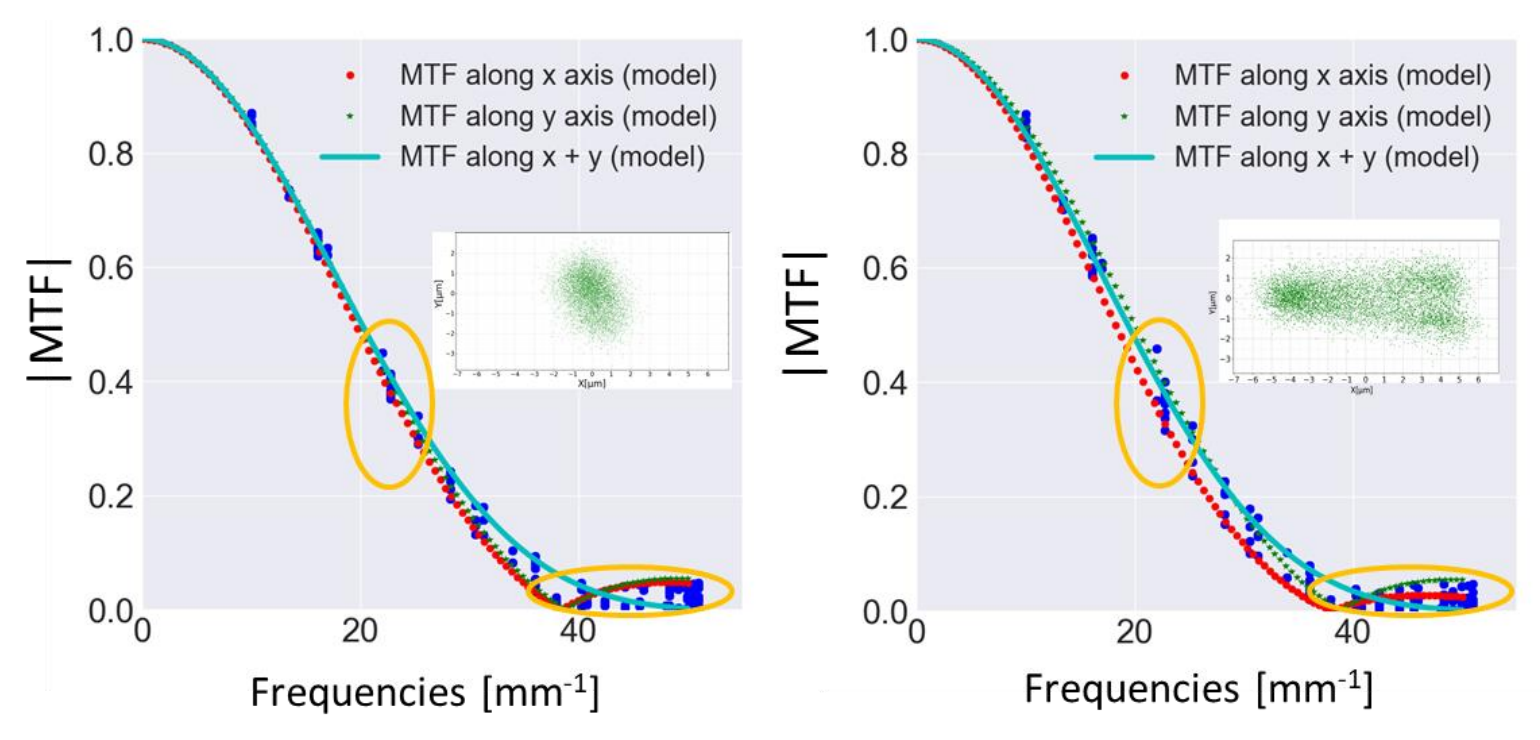

Figure 5 - MTF comparison between the optimized configuration and the one with an addtional $3 \mathrm{~kg}$ weight on the cryocooler.

\section{b) Analysis using accelerometers}

Accelerometers have been used to further comprehend the impact of the vibrations, their location on the bench being displayed in Figure 6. Accelerometer measurements on the compressor are reported in Figure 6 . A peak at $f_{\text {vib }}=31 \mathrm{~Hz}$ and its harmonics can be seen and has to be studied. The $31 \mathrm{~Hz}$ frequency has first to be compared to $\frac{1}{2 t_{i}}=82 \mathrm{~Hz}$, as it represents the actual temporal bandwidth of the measurement. 


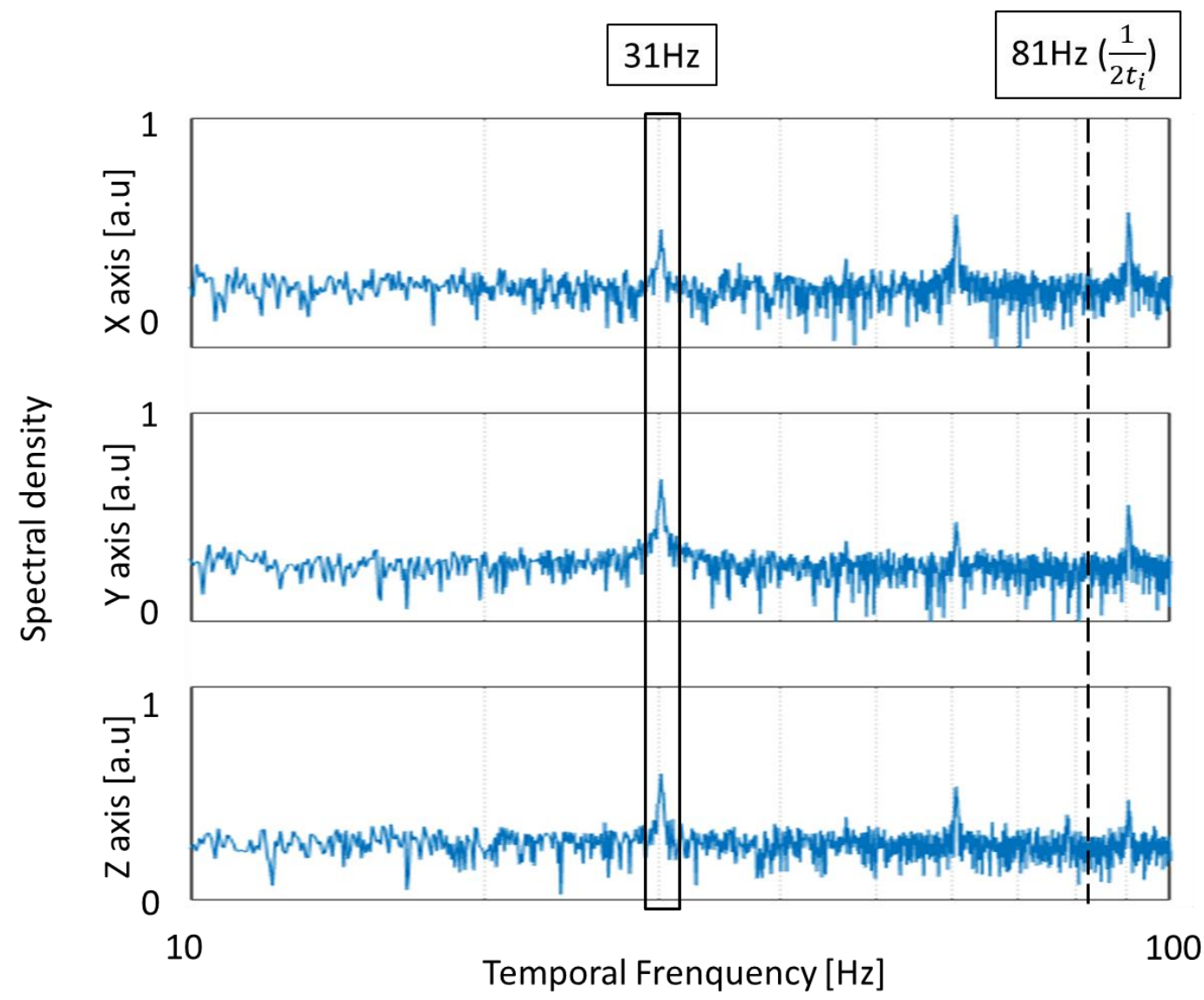

Figure 6 - Accelerometer data on the compressor (in logscale). One can see a peak at $31 \mathrm{~Hz}$ and its harmonics.

As in IDDCA configuration, data acquisition depends on the framerate and the integration time, both have to be taken into account in the analysis. As $f_{\text {vib }}$ is in the same order of magnitude as $\frac{1}{2 t_{i}}\left(\mathrm{t}_{\mathrm{i}}=6 \mathrm{~ms}\right.$ here), it means that there may be blur within images and between consecutive images. Let us considers that the movement of the FPA can be viewed as a sinus function. During $t_{i}$ in our case, the FPA will not be able to travel at least one full period of the sinusoid. However, it will have travelled too much distance to legitimate the hypothesis that the FPA did not move during $t_{i}$ as shown in Figure 7. It means that there will be a blur in the individual image. On top of that, the framerate $(60 \mathrm{~Hz}$ in our case) makes it less likely that by averaging several images, the same distance will be covered in each individual image or even the same part of the sinusoid, leading to a mix of images in which some individual images will be blurrier, while other are sharper. 


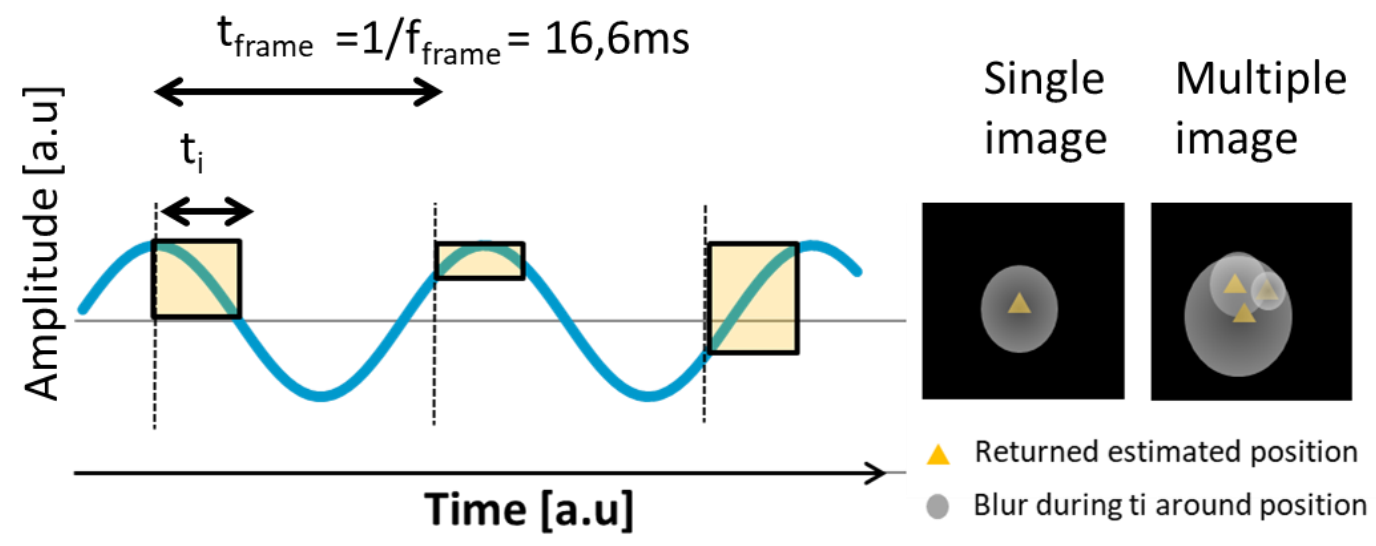

Figure 7 - Vibration analysis based on the vibration frequency versus the integration time $\mathrm{t}_{\mathrm{i}}$

Our approach is to estimate the worst impact that the $31 \mathrm{~Hz}$ vibration can have on the measurement. We identified the 2 extreme scenarii in Figure 8 . The first is that the FPA moved at maximum speed during the full integration time and will be called case 1 . The amplitude of the displacement then corresponds to the half amplitude of the sinusoid function. The other is when the two consecutive images are sharp, meaning that the amplitude found in Figure 4 corresponds to a shift between images and will be called case 2 . We consider that the amplitude $A=1.2 \mu \mathrm{m}$ found in Figure 4 is a good estimation of the amplitude of displacement for our vibration study.

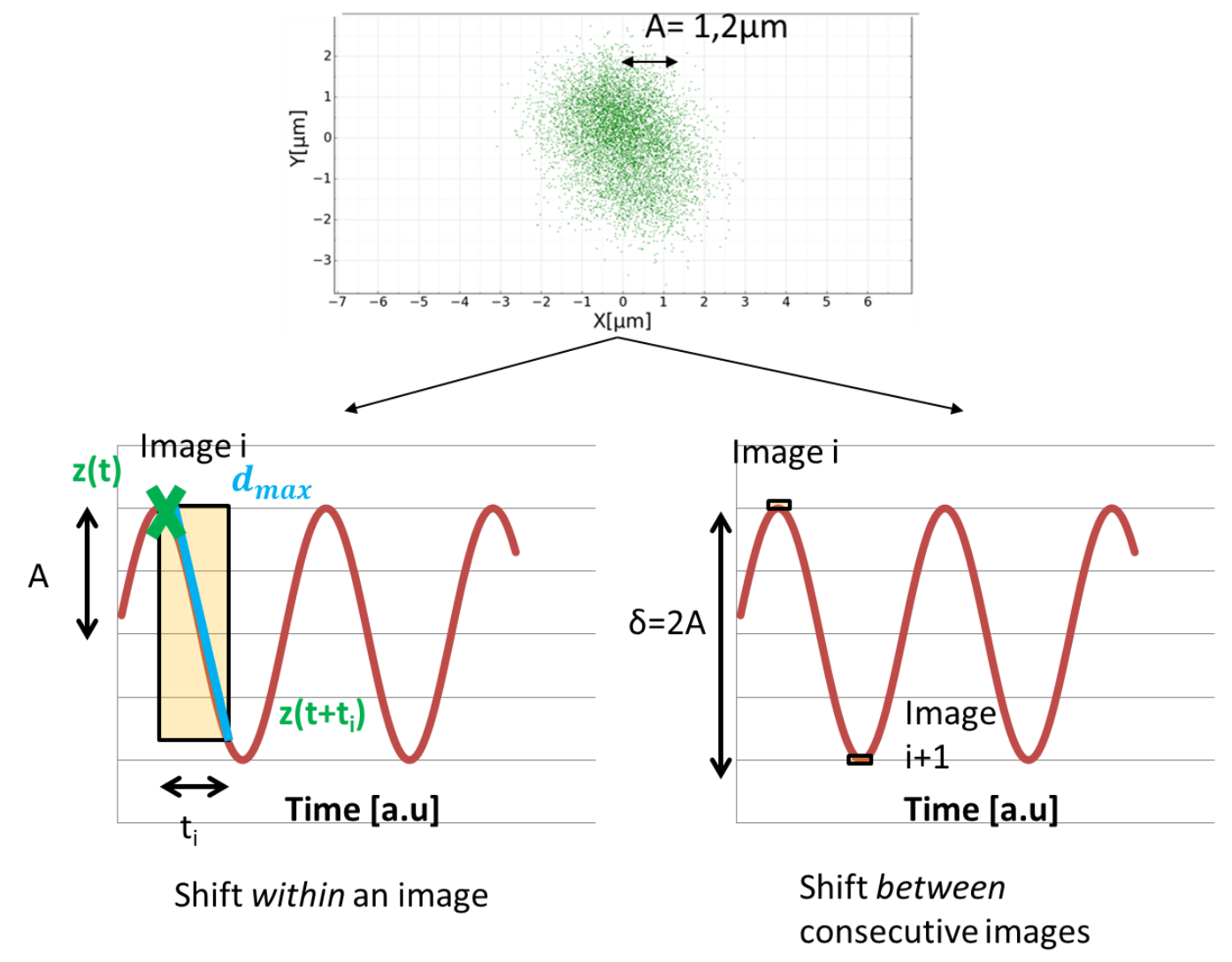

Figure 8 - The two extreme scenarii for the $31 \mathrm{~Hz}$ vibration. Either the FPA made the maximum displacement possible with a single image or the shift is between images. 
In case 1 , be $z(t)$ the position of the FPA. It can be written as :

$$
z(t)=A \sin \left(2 \pi t f_{v i b}\right)
$$

Maximum displacement $d_{\max }$ is obtained by calculating the maximum of the derivative of $z(t)$ and multiplying by $t_{i}$. It gives $d_{\max }=1.4 \mu m$ with $A=1.2 \mu m, t_{i}=6 m s$ and $f_{v i b}=31 \mathrm{~Hz}$.

The impact of $d_{\max }$ has to be compared to the pixel filtering effect. A blur of $d_{\max }$ is similar to a convolution of an image by a gate function of $d_{\max }$ width. It corresponds to a sinc in the fourier space, that is to say that we need to calculate :

$$
\operatorname{MTF}_{\text {blur }}(v)=\left|\operatorname{sinc}\left(v, d_{\text {max }}\right)\right|
$$

Nyquist frequency corresponds to the maximum spatial frequency properly sampled by the FPA and is defined as :

$$
f_{N y q}=\frac{1}{2 p}
$$

With $p$ the pixel pitch (here $p=30 \mu m$ ).

We have at Nyquist frequency :

$$
\operatorname{MTF} F_{b l u r}\left(f_{N y q}, d_{\text {max }}\right)=0.997
$$

And

$$
M T F_{\text {idealpixel }}\left(f_{N y q}, a_{\text {pix }}\right)=0.72
$$

With:

$$
M T F_{\text {idealpixel }}(f, a)=\operatorname{sinc}(f, a)
$$

As $M_{T F}$ blur is close to 1 and far higher than $M_{T F}$ idealpixel at Nyquist frequency, we can conclude that in case 1 , the vibration does not affect the MTF significantly.

In case 2, we can compare the shift of $2.4 \mu \mathrm{m}$ (twice the amplitude found in Figure 4) to a line of sight stabilization residual. The usual acceptable boundary being half a pixel $(13 \mu \mathrm{m}$ in our case), we can conclude that such shift can be compensated in operational systems thus will not have a significant impact on the MTF. 


\section{c) Perspective}

We conclude this vibration study that vibration cannot single-handedly explain the $\delta=10 \mu \mathrm{m}$ parameters found in section II (as the maximum we found for vibrations here is $2.4 \mu \mathrm{m}$ amplitude). This means that there is another filtering effect in mesa configuration, since the T2SL is deep-etched. Models are under development and will be the focus of future works.

\section{IV) Conclusion}

We report an MTF measurement on an operational T2SL MWIR FPA sealed in a cryocooler (IDDCA configuration). Our measurement bench grants access the phase of the MTF. It allows deducing a key detector parameter, the pixel size $(26 \mu \mathrm{m}$, confirmed by IRnova), without opening the IDDCA. In order to have good agreement between our model and experimental result, we added a 2D Gaussian with $\delta x$ dy parameters of $10 \mu \mathrm{m}$. As the T2SL studied has been deep etched, diffusion cannot explain these parameters. We then studied the impact of the vibration in our measurement, as it could explain this filtering effect. A study has been conducted with accelerometers. In our case, a $31 \mathrm{~Hz}$ vibration, within the same order of magnitude of $\frac{1}{2 t_{i}}$ has been detected during our MTF measurement. The upper boundary of the effect on the MTF has been determined and has shown to be negligible in the optimized bench compared to the pixel filtering or the line of sight stabilization, one can consider that this vibration does not deteriorate the MTF significantly. This means that a filtering effect exists in mesa configuration that has to be investigated in future works

\section{V) Funding}

Labex FOCUS (ANR-11-LABX-0013).

\section{VI) Reference}

[1] L. Höglund, C. Asplund, R. Marcks von Würtemberg, H.Kataria, A. Gamfeldt, S. Smuk, H. Martijn, and E. Costard, Manufacturability of type-II InAs/GaSb superlattice detectors for infrared imaging, Infrared Phys. Technol. 84(6) (2017) 28-32.

[2] A. Rogalski, M. Kopytko, P. Martyniuk, InAs/GaSb type-II superlattice infrared detectors: three decades of development, Proc. SPIE 10177 (2017) 1017715

[3] M. Delmas, R. Rossignol, J. B. Rodriguez, and P. Christol, Design of InAs/GaSb superlattice infrared barrier detectors, Superlattice. Microstruct. 104 (2017) 402-414. 
[4] D.Z. Ting, A. Soibel, A. Khoshakhlagh, L. Höglund, S.A. Keo, S.B. Rafol, C.J. Hill, A.M. Fisher, E.M. Luong, J. Nguyen, J.K. Liu, J.M. Mumolo, B.J. Pepper, and S.D. Gunapala, Antimonide type-II superlattice barrier infrared detectors, Proc. SPIE 10177 (2017) 101770N [5] P. C. Klipstein, Y. Livneh, A. Glozman, S. Grossman, O. Klin, N. Snapi, and E. Weiss, Modeling InAs/GaSb and InAs/InAsSb Superlattice Infrared Detectors, J. Electron. Mater. 43(8) (2014) 2984-2990.

[6] E. Giard, I. Ribet-Mohamed, J. Jaeck, T. Viale, R. Haïdar, R. Taalat, M. Delmas, J.-B. Rodriguez, E. Steveler, N. Bardou, F. Boulard, and P. Christol, "Quantum efficiency investigations of type-II InAs/GaSb midwave infrared superlattice photodetectors," J. Appl. Phys. 116(4) (2014) 043101

[7] M. Razeghi, A. Haddadi, A. M. Hoang, G. Chen, S. Bogdanov, S. R. Darvish, F. Callewaert, P. R. Bijjam, and R. McClintock, "Antimonide-Based Type II Superlattices: A Superior Candidate for the Third Generation of Infrared Imaging Systems," J. Electron. Mater. 43 (2014) 2802-2807

[8] R. Rehm, M. Walther, J. Schmitz, F. Rutz, J. Fleißner, R. Scheibner, and J. Ziegler, "InAs/GaSb superlattices for advanced infrared focal plane arrays," Infrared Phys. Technol. 52(6) (2009) 344-347.

[9] J. Nghiem, E. Giard, M. Delmas, J.B. Rodriguez, P. Christol, M. Caes, H. Martijn, E. Costard, I. Ribet-Mohamed, Radiometric characterization of type-II InAs/GaSb superlattice (T2SL) midwave infrared photodetectors and focal plane arrays, International Conference on Space Optics 10562 (2016) 105623Y

[10] I. Ribet-Mohamed, J. Nghiem, M. Caes, M. Guénin, L. Höglund, E. Costard, J.B. Rodriguez, P. Christol, Temporal stability and correctability of a MWIR T2SL focal plane array, Infrared Phys. Technol.

[11] N. Guérineau and J. Primot, Nondiffracting array generation using an N-wave interferometer. JOSA A, 16(2) (1999) 293-298.

[12] N. Guérineau, B. Harchaoui, J. Primot, , K.Heggarty, Generation of achromatic and propagation-invariant spot arrays by use of continuously self-imaging gratings. Optics letters, 26(7) (2001) 411-413.

[13] E. Di Mambro, N. Guérineau and J. Primot, Modulation transfer function measurement of an infrared focal plane array using a continuously self-imaging grating, Infrared Imaging Systems: Design, Analysis, Modeling, and Testing XIV 5076 (2003) 169-179.

[14] M. Piponnier, G. Druart, N. Guerineau, J. L. de Bougrenet and J. Primot : Optimal conditions for using the binary approximation of continuously self-imaging gratings. Opt.Express, 19 (2011) 23054-23066.

[15] F. de La Barrière, G. Druart, N.Guérineau, , S.Rommeluère, L., Mugnier , O. Gravrand, N. Baier, N. Lhermet, G. Destefanis and S. Derelle,. "Modulation Transfer Function Measurement of Infrared Focal-Plane Arrays with Small Fill Factors" , J. electron. mat., 41(10) (2012) 2730-2737

[16] G. Druart, N. Guérineau, R.Haïdar, J.Primot, P. Chavel, and J. Taboury, Nonparaxial analysis of continuous self-imaging gratings in oblique illumination, JOSA A, 24(10) (2007) 3379-3387.

[17] G. D. Boreman, Modulation transfer function in optical and electro-optical systems SPIE press. 21 (2001) https://spie.org/Publications/Book/419857 